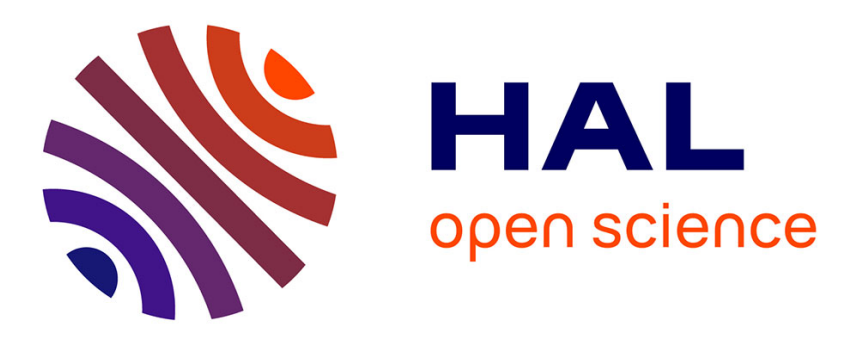

\title{
A Highly Scalable Parallel Algorithm for Maximally Informative k-Itemset Mining
}

Saber Salah, Reza Akbarinia, Florent Masseglia

\section{To cite this version:}

Saber Salah, Reza Akbarinia, Florent Masseglia. A Highly Scalable Parallel Algorithm for Maximally Informative k-Itemset Mining. Knowledge and Information Systems (KAIS), 2017, 50 (1), pp.1-26. 10.1007/s10115-016-0931-2 . lirmm-01288571

\section{HAL Id: lirmm-01288571 https://hal-lirmm.ccsd.cnrs.fr/lirmm-01288571}

Submitted on 15 Mar 2016

HAL is a multi-disciplinary open access archive for the deposit and dissemination of scientific research documents, whether they are published or not. The documents may come from teaching and research institutions in France or abroad, or from public or private research centers.
L'archive ouverte pluridisciplinaire HAL, est destinée au dépôt et à la diffusion de documents scientifiques de niveau recherche, publiés ou non, émanant des établissements d'enseignement et de recherche français ou étrangers, des laboratoires publics ou privés. 


\title{
A Highly Scalable Parallel Algorithm for Maximally Informative k-Itemset Mining
}

\author{
Saber Salah, Reza Akbarinia and Florent Masseglia \\ Inria and Lirmm, Montpellier, France
}

\begin{abstract}
.
The discovery of informative itemsets is a fundamental building block in data analytics and information retrieval. While the problem has been widely studied, only few solutions scale. This is particularly the case when i) the data set is massive, calling for large-scale distribution, and/or ii) the length $k$ of the informative itemset to be discovered is high. In this paper, we address the problem of parallel mining of maximally informative $k$-itemsets (miki) based on joint entropy. We propose PHIKS (Parallel Highly Informative $\underline{K}$-ItemSet) a highly scalable, parallel miki mining algorithm. PHIK $\bar{S}$ renders the mining process of large scale databases (up to terabytes of data) succinct and effective. Its mining process is made up of only two efficient parallel jobs. With PHIKS, we provide a set of significant optimizations for calculating the joint entropies of miki having different sizes, which drastically reduces the execution time, the communication cost and the energy consumption, in a distributed computational platform. PHIKS has been extensively evaluated using massive real-world data sets. Our experimental results confirm the effectiveness of our proposal by the significant scale-up obtained with high itemsets length and over very large databases.
\end{abstract}

Keywords: Joint entropy; Informative itemsets; Massive distribution; MapReduce; Spark; Hadoop Big Data

\section{Introduction}

Featureset, or itemset, mining (Han, 2012) is one of the fundamental building bricks for exploring informative patterns in databases. Features might be, for instance, the words occurring in a document, the score given by a user to a movie on a social network, or the characteristics of plants (growth, genotype, humidity, biomass, etc.) in a scientific study in agronomics. A large number of contributions in the literature has been proposed for itemset mining, exploring various measures according to the chosen relevance criteria. The most studied measure is probably the number of co-occurrences of a set of features, also known as frequent itemsets (Agrawal and Srikant, 1994). However, frequency does not give relevant results for a various range of applications, including information retrieval (Greengrass, 2000), since it does not give a complete overview 


\begin{tabular}{|c|c|c|c|c|c|c|c|c|c|c|}
\hline \multirow[t]{2}{*}{ Features } & \multicolumn{10}{|c|}{ Documents } \\
\hline & $d_{1}$ & $d_{2}$ & $d_{3}$ & $d_{4}$ & $d_{5}$ & $d_{6}$ & $d_{7}$ & $d_{8}$ & $d_{9}$ & $d_{10}$ \\
\hline A & 1 & 1 & 1 & 1 & 1 & 0 & 0 & 0 & 0 & 0 \\
\hline B & 0 & 1 & 0 & 0 & 1 & 1 & 0 & 1 & 0 & 1 \\
\hline $\mathrm{C}$ & 1 & 0 & 0 & 1 & 0 & 1 & 1 & 0 & 1 & 0 \\
\hline $\mathrm{D}$ & 1 & 0 & 1 & 1 & 1 & 1 & 1 & 1 & 1 & 1 \\
\hline E & 1 & 1 & 1 & 1 & 1 & 1 & 1 & 1 & 1 & 1 \\
\hline
\end{tabular}

Table 1. Features in The Documents

of the hidden correlations between the itemsets in the database. This is particularly the case when the database is sparse (Heikinheimo, Hinkkanen, Mannila, Mielikäinen and Seppänen, 2007). Using other criteria to assess the informativeness of an itemset could result in discovering interesting new patterns that were not previously known. To this end, information theory (Cover, 2006) gives us strong supports for measuring the informativeness of itemsets. One of the most popular measures is the joint entropy (Cover, 2006) of an itemset. An itemset $X$ that has higher joint entropy brings up more information about the objects in the database.

We study the problem of Maximally Informative $k$-Itemsets (miki for short) discovery in massive data sets, where informativeness is expressed by means of joint entropy and $k$ is the size of the itemset (Gray, 2011; Knobbe and Ho, 2006; Zhang and Masseglia, 2010). Miki are itemsets of interest that better explain the correlations and relationships in the data. Example 1 gives an illustration of miki and its potential for real world applications such as information retrieval.

Example 1. In this application, we would like to retrieve documents from Table 1, in which the columns $d_{1}, d_{10}$ are documents, and the attributes $A, B, C, D, E$ are some features (items, keywords) in the documents. The value " 1 " means that the feature occurs in the document, and " 0 " not. It is easy to observe that the itemset $(D, E)$ is frequent, because features $D$ and $E$ occur together in almost every document. However, it provides little help for document retrieval. In other words, given a document $d_{x}$ in our data set, one might look for the occurrence of the itemset $(D, E)$ and, depending on whether it occurs or not, she will not be able to decide which document it is. By contrast, the itemset $(A, B, C)$ is infrequent, as its member features rarely or never appear together in the data. And it is troublesome to summarize the value patterns of the itemset $(A, B, C)$. Providing it with the values $<1,0,0>$ we could find the corresponding document $O_{3}$; similarly, given the values $<0,1,1>$ we will have the corresponding document $O_{6}$. Although $(A, B, C)$ is infrequent, it contains lots of useful information which is hard to summarize. By looking at the values of each feature in the itemset $(A, B, C)$, it is much easier to decide exactly which document they belong to. $(A, B, C)$ is a maximally informative itemset of size $k=3$.

Miki mining is a key problem in data analytics with high potential impact on various tasks such as supervised learning (Kotsiantis, 2007), unsupervised learning (Ghahramani, 2004) or information retrieval (Greengrass, 2000), to cite a few. A typical application is the discovery of discriminative sets of features, based on joint entropy (Cover, 2006), which allows distinguishing between different categories of objects. Unfortunately, it is very difficult to maintain good results, in terms of both response time and quality, when the number of objects becomes very large. Indeed, with massive amounts of data, 
computing the joint entropies of all itemsets in parallel is a very challenging task for many reasons. First, the data is no longer located in one computer, instead, it is distributed over several machines. Second, the number of iterations of parallel jobs would be linear to $k$ (i.e., the number of features in the itemset to be extracted (Knobbe and Ho, 2006)), which needs multiple database scans and in turn violates the parallel execution of the mining process. We believe that an efficient miki mining solution should scale up with the increase in the size of the itemsets, calling for cutting edge parallel algorithms and high performance evaluation of an itemset's joint entropy in massively distributed environments.

We propose a deep combination of both information theory and massive distribution by taking advantage of parallel programming frameworks such as MapReduce (Dean and Ghemawat, 2008) or Spark (Zaharia, Chowdhury, Franklin, Shenker and Stoica, 2010). To the best of our knowledge, there has been no prior work on parallel informative itemsets discovery based on joint entropy. We designed and developed an efficient parallel algorithm, namely Parallel Highly Informative $K$-itemSet (PHIKS in short), that renders the discovery of miki from a very large database (up to Terabytes of data) simple and effective. It performs the mining of miki in two parallel jobs. PHIKS cleverly exploits available data at each mapper to efficiently calculate the joint entropies of miki candidates. For more efficiency, we provide PHIKS with optimizations that allow for very significant improvements of the whole process of miki mining. The first technique estimates the upper bound of a given set of candidates and allows for a dramatic reduction of data communications, by filtering unpromising itemsets without having to perform any additional scan over the data. The second technique reduces significantly the number of scans over the input database of each mapper, i.e., only one scan per step, by incrementally computing the joint entropy of candidate features. This reduces drastically the work that should be done by the mappers, and thereby the total execution time.

PHIKS has been extensively evaluated using massive real-world data sets. Our experimental results show that PHIKS significantly outperforms alternative approaches, and confirm the effectiveness of our proposal over large databases containing for example one Terabyte of data.

The rest of the paper is structured as follows. Section 2 discusses related work. Section 3 gives formal definitions of informative itemsets, basic used notations, and the necessary background is given in Section 4 . In Section 5 we propose our PHIKS algorithm, and depict its whole core mining process. Section 6 reports on our experimental validation over real-world data sets. and Section 7 concludes.

\section{Related Work}

In data mining literature, several endeavors have been made to explore informative itemsets (or featuresets, or set of attributes) in databases (Agrawal and Srikant, 1994) (Han, Pei and Yin, 2000) (Heikinheimo et al., 2007) (Knobbe and Ho, 2006). Different measures of itemset informativeness (e.g., frequency of itemset co-occurrence in the database etc.) have been used to identify and distinguish informative itemsets from non-informative ones. For instance, by considering the itemsets co-occurrence, several conclusions can be drawn to explain interesting, hidden relationships between different itemsets in the data.

Mining itemsets based on the co-occurrence frequency (e.g., frequent itemset mining) measure does not capture all dependencies and hidden relationships in the database, especially when the data is sparse (Heikinheimo et al., 2007). Therefore, other measures 
must be taken into account. Low and high entropy measures of itemsets informativeness were proposed (Heikinheimo et al., 2007). The authors of (Heikinheimo et al., 2007) have proposed the use of a tree based structure without specifying a length $k$ of the informative itemsets to be discovered. However, as the authors of (Heikinheimo et al., 2007) mentioned, such an approach results in a very large output.

Beyond using a regular co-occurrence frequency measure to identify the itemsets informativeness, the authors of (Brin, Motwani and Silverstein, 1997) have proposed an efficient technique that is more general. The main motivation is to get better insight and understanding of the data by figuring out other hidden relationships between the itemsets (i.e., the inner correlation between the itemsets themselves), in particular when determining the itemsets' rules. To this end, the authors of (Brin et al., 1997) did not focus only on the analysis of the positive implications between the itemsets in the data (i.e., implications between supported itemsets), but also they take into account the negative implications. To determine the significance of such itemsets implications, the authors of (Brin et al., 1997) have used a classic statistical chi-squared measure to efficiently figure out the interestingness of such itemsets rules.

Generally, in the itemset mining problem there is a trade-off between the itemset informativeness and the pattern explosion (i.e., number of itemsets to be computed). Thus, some itemset informativeness measures (e.g., the co-occurrence frequency measure with very low minimum support) would allow for a potential high number of useless patterns (i.e., itemsets), and others would highly limit the number of patterns. The authors of (Tatti, 2010) proposed an efficient approach that goes over regular used itemset informativeness measures, by developing a general framework of statistical models allowing the scoring of the itemsets in order to determine their informativeness. In particular, in (Tatti, 2010), the initial focus is on the exponential models to score the itemsets. However these models are inefficient in terms of execution time, thus, the authors propose to use decomposable models. On the whole, the techniques proposed in (Tatti, 2010) and (Brin et al., 1997) are mainly dedicated to mining in centralized environments, while our techniques are dedicated to parallel data mining in distributed environments.

The authors of (Knobbe and Ho, 2006) suggest to use a heuristic approach to extract informative itemsets of length $k$ based on maximum joint entropy. Such maximally informative itemsets of size $k$ is called miki. This approach captures the itemsets that have high joint entropies. An itemset is a miki if all of its constructing items shatter the data maximally. The items within a miki are not excluding, and do not depend on each other. (Knobbe and Ho, 2006) proposes a bunch of algorithms to extract miki. A brute force approach consists of performing an exhaustive search over the database to determine all miki of different sizes. However, this approach is not feasible due to the large number of itemsets to be determined, which results in multiple database scans. Another algorithm proposed in (Knobbe and Ho, 2006) consists of fixing a parameter $k$ that denotes the size of the miki to be discovered. This algorithm proceeds by determining a top $n$ miki of size 1 having highest joint entropies, then, the algorithm determines the combinations of 1 -miki of size 2 and returns the top $n$ most informative itemsets. The process continues until it returns the top $n$ miki of size $k$.

The problem of extracting informative itemsets was not only proposed for mining static databases. There have been also interesting works in extracting informative itemsets in data streams (Giannella, Han, Pei, Yan and Yu, 2002) (Teng, Chen and Yu, 2003). The authors of (Zhang and Masseglia, 2010) proposed an efficient method for discovering maximally informative itemsets (i.e., highly informative itemsets) from data streams based on sliding window.

Extracting informative itemsets has a prominent role in feature selection (Chandrashekar and Sahin, 2014). Various techniques and methods have been proposed 
in the literature to solve the problem of selecting relevant features to be used in classification tasks. These methods fall into two different categories, namely Filter and Wrapper methods (Guyon and Elisseeff, 2003). Filter methods serve to pre-process the data before being used for a learning purpose. They aim to determine a small set of relevant features. However, these methods capture only the correlations between each feature (i.e., independent variable, attribute or item) and the target class (i.e., predictor). They do not take into account the inter correlation between the selected features (i.e., if the features are inter correlated then they are redundant). In the other hand, to determine an optimal set of relevant features, wrapper methods perform a feature's set search that maximizes an objective function (i.e., classifier performance). However, these methods yield in heavy computations (i.e., selecting each time a set of features and evaluate an objective function). To solve this problem, Embedded (Chandrashekar and Sahin, 2014) methods have been proposed. The main goal is to incorporate the wrapper methods in the learning process.

Processing very large amount of data with high number of features (i.e., billions of items) to select most relevant once's is not trivial. Miki are very good challenging candidates to select relevant features in large-scale databases. The items (i.e., features) that a set of miki contains have very low inter correlations, thus they highly discriminate the whole database together. This property of miki make them of a highly potential success in improving different data mining tasks such as subgroup discovery (Herrera, Carmona, González and del Jesus, 2011) and classification (Berry and Castellanos, n.d.) problems etc.

Parallel mining of informative itemsets from large databases based on frequency informativeness measure has received much attention recently (Li, Wang, Zhang, Zhang and Chang, 2008) (Riondato, DeBrabant, Fonseca and Upfal, 2012) (Tanbeer, Ahmed and Jeong, 2009). For instance, In (Li et al., 2008), the authors have proposed an efficient parallel solution to extract frequent itemsets based on FP-Growth algorithm (Han et al., 2000). Their algorithm PFP-Growth has gained a popular success in mining large databases. In (Riondato et al., 2012), the authors have proposed an improvement of PFPGrowth algorithm by performing approximations of the candidate frequent itemsets.

To the best of our knowledge, there has been no prior work on parallel discovery of maximally informative $k$-itemsets in large databases.

\section{Definitions}

The following definitions introduce the basic requirements for mining maximally informative $k$-itemsets (Knobbe and Ho, 2006).

Definition 1. Let $\mathcal{F}=\left\{f_{1}, f_{2}, \ldots, f_{n}\right\}$ be a set of literals called features. An itemset $X$ is a set of features from $\mathcal{F}$, i.e., $X \subseteq \mathcal{F}$. The size or length of the itemset $X$ is the number of features in it. A transaction $T$ is a set of elements such that $T \subseteq \mathcal{F}$ and $T \neq \emptyset$. A database $\mathcal{D}$ is a set of transactions.

Definition 2. The entropy (Cover, 2006) of a feature $i$ in a database $\mathcal{D}$ measures the expected amount of information needed to specify the state of uncertainty or disorder for the feature $i$ in $\mathcal{D}$. Let $i$ be a feature in $\mathcal{D}$, and $P(i=n)$ be the probability that $i$ has value $n$ in $\mathcal{D}$ (we consider categorical data, where the value will be '1' if the object has the feature and ' 0 ' otherwise). The entropy of the feature $i$ is given by

$$
H(i)=-(P(i=0) \log (P(i=0))+P(i=1) \log (P(i=1)))
$$

where the logarithm base is 2 . 
Definition 3. The binary projection, or projection of an itemset $X$ in a transaction $T$ $(\operatorname{proj}(X, T))$ is the set of size $|X|$ where each item (i.e., feature) of $X$ is replaced by '1' if it occurs in $T$ and by ' 0 ' otherwise. The projection counting of $X$ in a database $\mathcal{D}$ is the set of projections of $X$ in each transaction of $\mathcal{D}$, where each projection is associated with its number of occurrences in $\mathcal{D}$.

Example 2. Let us consider Table 1. The projection of $(B, C, D)$ in $d_{1}$ is $(0,1,1)$. The projections of $(D, E)$ on the database of Table 1 are $(1,1)$ with nine occurrences and $(0,1)$ with one occurrence.

Definition 4. Given an itemset $X=\left\{x_{1}, x_{2}, \ldots, x_{k}\right\}$ and a tuple of binary values $\mathcal{B}=\left\{b_{1}, b_{2}, \ldots, b_{k}\right\} \in\{01\}^{k}$. The joint entropy of $X$ is defined as:

$$
H(X)=-\sum_{\mathcal{B} \in\{0,1\}^{|k|}} J \times \log (J)
$$

Where $J=P\left(x_{1}=b_{1}, \ldots, x_{k}=b_{k}\right)$ is the joint probability of $X=\left\{x_{1}, x_{2}, \ldots, x_{k}\right\}$.

Given a database $\mathcal{D}$, the joint entropy $H(X)$ of an itemset $X$ in $\mathcal{D}$ is proportional to its size $k$ i.e., the increase in the size of $X$ implies an increase in its joint entropy $H(X)$. The higher the value of $H(X)$, the more information the itemset $X$ provides in $\mathcal{D}$. For simplicity, we use the term entropy of an itemset $X$ to denote its joint entropy.

Example 3. Let us consider the database of Table 1 . The joint entropy of $(D, E)$ is given by $H(D, E)=-\frac{9}{10} \log \left(\frac{9}{10}\right)-\frac{1}{10} \log \left(\frac{1}{10}\right)=0.468$. Where the quantities $\frac{9}{10}$ and $\frac{1}{10}$ respectively represent the joint probabilities of the projection values $(1,1)$ and $(0,1)$ in the database.

Definition 5. Given a set $\mathcal{F}=\left\{f_{1}, f_{2}, \ldots, f_{n}\right\}$ of features, an itemset $X \subseteq \mathcal{F}$ of length $k$ is a maximally informative $k$-itemset, if for all itemsets $Y \subseteq \mathcal{F}$ of size $k$, $H(Y) \leq H(X)$. Hence, a maximally informative $k$-itemset is the itemset of size $k$ with the highest joint entropy value.

The problem of mining maximally informative $k$-itemsets presents a variant of itemset mining, it relies on the joint entropy measure for assessing the informativeness brought by an itemset.

Definition 6. Given a database $\mathcal{D}$ which consists of a set of $n$ attributes (features) $\mathcal{F}=\left\{f_{1}, f_{2}, \ldots, f_{n}\right\}$. Given a number $k$, the problem of miki mining is to return a subset $F^{\prime} \subseteq F$ with size $k$, i.e., $\left|F^{\prime}\right|=k$, having the highest joint entropy in $\mathcal{D}$, i.e., $\forall F^{\prime \prime} \subseteq F \wedge\left|F^{\prime \prime}\right|=k, H\left(F^{\prime \prime}\right) \leq H\left(F^{\prime}\right)$.

\section{Background}

In this Section, first we detail the miki discovery in a centralized environment. Second, we detail the working principle of MapReduce, in particular, we depict the execution process of a MapReduce job.

\subsection{Miki Discovery in a Centralized Environment}

In (Knobbe and Ho, 2006), an effective approach is proposed for miki discovery in a centralized environment. Their ForwardSelection heuristic uses a "generating-pruning" 
approach, which is similar to the principle of Apriori (Agrawal and Srikant, 1994). $i_{1}$, the feature having the highest entropy is selected as a seed. Then, $i_{1}$ is combined with all the remaining features, in order to build candidates. In other words, there will be $|\mathcal{F}-1|$ candidates $\left(i . e .,\left(i_{1}, i_{2}\right),\left(i_{1}, i_{3}\right), \ldots,\left(i_{1}, i_{|\mathcal{F}-1|}\right)\right)$. The entropy of each candidate is given by a scan over the database, and the candidate having the highest entropy, say $\left(i_{1}, i_{2}\right)$, is kept. A set of $|\mathcal{F}-2|$ candidates of size 3 is generated (i.e., $\left.\left(i_{1}, i_{2}, i_{3}\right),\left(i_{1}, i_{2}, i_{4}\right), \ldots,\left(i_{1}, i_{2}, i_{|\mathcal{F}-2|}\right)\right)$ and their entropy is given by a new scan over the database. This process is repeated until the size of the extracted itemset is $k$.

\subsection{MapReduce and Job Execution}

MapReduce has gained increasing popularity, as shown by the tremendous success of Hadoop (White, 2012), an open-source implementation. It is one of the most popular solutions for big data processing (Bizer, Boncz, Brodie and Erling, 2011), in particular due to its automatic management of parallel execution in clusters of machines. Initially proposed in (Dean and Ghemawat, 2008), it was popularized by Hadoop (Hadoop, 2014), an open-source implementation. MapReduce divides the computation in two phases, namely map and reduce, which in turn are carried out by several tasks that process the data in parallel. The idea behind MapReduce is simple and elegant. Given an input file, and two functions map and reduce, each MapReduce job is executed in two main phases: map and reduce. In the first phase, called map, the input data is divided into a set of splits, and each split is processed by a map task in a given worker node. These tasks apply the map function on every key-value pair of their split and generate a set of intermediate pairs. In the second phase, called reduce, all the values of each intermediate key are grouped and assigned to a reduce task. Reduce tasks are also assigned to worker machines and apply the reduce function on the created groups to produce the final results. Each MapReduce job includes two functions: map and reduce. For executing the job, we need a master node for coordinating the job execution, and some worker nodes for executing the map and reduce tasks. When a MapReduce job is submitted by a user to the cluster, after checking the input parameters, e.g., input and output directories, the input splits (blocks) are computed. The number of input splits can be personalized, but typically there is one split for each $64 \mathrm{MB}$ of data. The location of these splits and some information about the job are submitted to the master. The master creates a job object with all the necessary information, including the map and reduce tasks to be executed. One map task is created per input split. When a worker node, say $w$, becomes idle, the master tries to assign a task to it. The map tasks are scheduled using a locality-aware strategy. Thus, if there is a map task whose input data is kept on $w$, then the scheduler assigns that task to $w$. If there is no such task, the scheduler tries to assign a task whose data is in the same rack as $w$ (if any). Otherwise, it chooses any task. Each map task reads its corresponding input split, applies the map function on each input pair and generates intermediate key-value pairs. , which are firstly maintained in a buffer in main memory. When the content of the buffer reaches a threshold (by default $80 \%$ of its size), the buffered data is stored on the disk in a file called spill. Once the map task is completed, the master is notified about the location of the generated intermediate key-values. In the reduce phase, each intermediate key is assigned to one of the reduce workers. Each reduce worker retrieves the values corresponding to its assigned keys from all the map workers, and merges them using an external merge-sort. Then, it groups pairs with the same key and calls the reduce function on the corresponding values. This function will generate the final output results. When, all tasks of a job are completed successfully, the client is notified by the master. 


\section{PHIKS Algorithm}

In a massively distributed environment, a possible naive approach for miki mining would be a straightforward implementation of ForwardSelection (Knobbe and Ho, 2006) (see Section 4.1). However, given the "generating-pruning" principle of this heuristic, it is not suited for environments like Spark (Zaharia et al., 2010) or MapReduce (Dean and Ghemawat, 2008) and would lead to very bad performances. The main reason is that each scan over the data set is done through a distributed job (1.e., there will be $k$ jobs, one for each generation of candidates that must be tested over the database). Our experiments, in Section 6, give an illustration of the catastrophic response times of ForwardSelection in a straightforward implementation on MapReduce (the worst, for all of our settings). This is not surprising since most algorithms designed for a centralized itemset mining do not perform well in massively distributed environments in a direct implementation (Moens, Aksehirli and Goethals, 2013), (Berberich and Bedathur, 2013), (Miliaraki, Berberich, Gemulla and Zoupanos, 2013), and miki don't escape that rule.

Such an inadequacy calls for new distributed algorithmic principles. To the best of our knowledge, there is no previous work on distributed mining of miki. However, we may build on top of cutting edge studies in frequent itemset mining, while considering the very demanding characteristics of miki.

Interestingly, in the case of frequent itemsets in MapReduce, a mere algorithm consisting of two jobs outperforms most existing solutions (Anand, 2012) by using the principle of SON (Savasere, Omiecinski and Navathe, 1995), a divide and conquer algorithm. Unfortunately, despite its similarities with frequent itemset mining, the discovery of miki is much more challenging. Indeed, the number of occurrences of an itemset $X$ in a database $\mathcal{D}$ is additive and can be easily distributed (the global number of occurrences of $X$ is simply the sum of its local numbers of occurrences on subsets of $\mathcal{D}$ ). Entropy is much more combinatorial since it is based on the projection counting of $X$ in $\mathcal{D}$ and calls for efficient algorithmic advances, deeply combined with the principles of distributed environments.

\subsection{Distributed Projection Counting}

Before presenting the details of our contribution, we need to provide tools for computing the projection of an itemset $X$ on a database $\mathcal{D}$, when $\mathcal{D}$ is divided into subsets on different splits, in a distributed environment, and entropy has to be encoded in the keyvalue format. We have to count, for each projection $p$ of $X$, its number of occurrences on $\mathcal{D}$. This can be solved with an association of the itemset as a key and the projection as a value. On a split, for each projection of an itemset $X, X$ is sent to the reducer as the key coupled with its projection. The reducer then counts the number of occurrences, on all the splits, of each (key:value) couple and is therefore able to calculate the entropy of each itemset. Communications may be optimized by avoiding to emit a key : val couple when the projection does not appear in the transaction and is only made of ' 0 ' (on the reducer, the number of times that a projection $p$ of $X$ does not appear in $\mathcal{D}$ is determined by subtracting the number projections of $X$ in $D$ from $|\mathcal{D}|$ ).

Example 4. Let us consider $\mathcal{D}$, the database of Table 1 and the itemset $X=(D, E)$. Let us consider that $\mathcal{D}$ is divided into two splits $S_{1}=\left\{d_{1} . . d 5\right\}$ and $S_{2}=\left\{d_{6} . . d_{10}\right\}$. With one simple MapReduce job, it is possible to calculate the entropy of $X$. The algorithm of a mapper would be the following: for each document $d$, emit a couple (key : val) where key $=X$ and $v a l=\operatorname{proj}(X, d)$. The first mapper (corresponding 
to $\left.S_{1}\right)$ will emit the following couples: $((D, E):(1,1)) 4$ times and $((D, E):(0,1))$ once. The second mapper will emit $((D, E):(1,1)) 5$ times. The reducers will do the sum and the final result will be $((D, E):(1,1))$ occurs 9 times and $(((D, E):(0,1))$ once.

\subsection{Discovering miki in Two Rounds}

Our heuristic will use at most two MapReduce jobs in order to discover the $k$-itemset having the highest entropy. The goal of the first job is to extract locally, on the distributed subsets of $\mathcal{D}$, a set of candidate itemsets that are likely to have a high global entropy. To that end, we apply the principle of ForwardSelection locally, on each mapper, and grow an itemset by adding a new feature at each step. After the last scan, for each candidate itemset $X$ of size $k$ we have the projection counting of $X$ on the local data set. A straightforward approach would be to emit the candidate itemset having the highest local entropy. We denote by local entropy, the entropy of an itemset in a subset of the database that is read by a mapper (i.e., by considering only the projections of $X$ in the mapper). Then the reducers would collect the local miki and we would check their global entropy (i.e., the entropy of the itemset $X$ in the entire database $\mathcal{D}$ ) by means of a second MapReduce job. Unfortunately, this approach would not be correct, since an itemset might have the highest global entropy, while actually not having the highest entropy in each subset. Example 5 gives a possible case where a global miki does not appear as a local miki on any subset of the database.

Example 5. Let us consider $\mathcal{D}$, the database given by Table 2 , which is divided into two splits of six transactions. The global miki of size 3 in this database is $(A, B, E)$. More precisely, the entropy of $(A, B, E)$ on $\mathcal{D}$ is given by $-\frac{1}{12} \times \log \left(\frac{1}{12}\right) \times 4-\frac{2}{12} \times$ $\log \left(\frac{2}{12}\right) \times 4=2.92$. However, if we consider each split individually, $(A, B, E)$ always has a lower entropy compared to at least one different itemset. For instance, on the split $S_{1}$, the projections of $(A, B, E)$ are $(0,0,0),(0,1,0),(1,1,0)$ and $(0,1,1)$ with one occurrence each, and $(1,0,0)$ with two occurrences. Therefore the entropy of $(A, B, E)$ on $S_{1}$ is 2.25 (i.e., $\left.-\frac{1}{6} \times \log \left(\frac{1}{6}\right) \times 4-\frac{2}{6} \times \log \left(\frac{2}{6}\right)=2.25\right)$. On the other hand, the projections of $(A, B, C)$ on $S_{1}$ are $(0,0,1),(0,1,0),(1,0,1),(1,1,0),(0,1,1)$ and $(1,0,0)$ with one occurrence each, and the entropy of $(A, B, C)$ on $S_{1}$ is 2.58 (i.e., $\left.-\frac{1}{6} \times \log \left(\frac{1}{6}\right) \times 6=2.58\right)$. This is similar on $S_{2}$ where the entropy of $(A, B, E)$ is 2.25 and the entropy of $(A, B, D)$ is 2.58 . However, $(A, B, C)$ and $(A, B, D)$ both have a global entropy of 2.62 on $\mathcal{D}$, which is lower than 2.92 , the global entropy of $(A, B, E)$ on $\mathcal{D}$.

Since it is possible that a global miki is never found as a local miki, we need to consider a larger number of candidate itemsets. This can be done by exploiting the set of candidates that are built in the very last step of ForwardSelection. This step aims to calculate the projection counting of $\mathcal{F}-k$ candidates and then compute their local entropy. Instead of only emitting the itemset having the larger entropy, we will emit, for each candidate $X$, the projection counting of $X$ on the split, as explained in Section 5.1. The reducers will then be provided with, for each local candidate $X_{i}(1 \leq i \leq m$, where $m$ is the number of mappers, or splits), the projection counting of $X$ on a subset of $\mathcal{D}$. The main idea is that the itemset having the highest entropy is highly likely to be in that set of candidates. For instance, in the database given by Table 2 and $k=3$, the global miki is $(A, B, E)$, while the local miki are $(A, B, C)$ on $S_{1}$ and $(A, B, D)$ on $S_{2}$. However, with the technique described above, the itemset $(A, B, E)$ will be a local candidate, and will be sent to the reducers with the whole set of projections encountered 


\begin{tabular}{cccccc}
\hline Split & A & B & C & D & E \\
\hline$S_{1}$ & 0 & 0 & 1 & 0 & 0 \\
& 0 & 1 & 0 & 0 & 0 \\
& 1 & 0 & 1 & 0 & 0 \\
& 1 & 1 & 0 & 0 & 0 \\
& 0 & 1 & 1 & 0 & 1 \\
& 1 & 0 & 0 & 0 & 0 \\
\hline$S_{2}$ & 0 & 0 & 0 & 0 & 1 \\
& 0 & 1 & 0 & 1 & 1 \\
& 1 & 0 & 0 & 0 & 1 \\
& 1 & 1 & 0 & 1 & 1 \\
& 0 & 1 & 0 & 0 & 0 \\
& 1 & 0 & 0 & 1 & 1
\end{tabular}

Table 2. Local Vs. Global Entropy

so far in the splits. The reducer will then calculate its global entropy, compare it to the entropy of the other itemsets, and $(A, B, E)$ will eventually be selected as the miki on this database.

Unfortunately, it is possible that $X$ has not been generated as a candidate itemset on the entire set of splits (consider a biased data distribution, where a split contains some features with high entropies, and these features have low entropies on the other splits). Therefore, we have two possible cases at this step:

1. $X$ is a candidate itemset on all the splits and we are able to calculate its exact projection counting on $\mathcal{D}$, by means of the technique given in Section 5.1 .

2. There is (at least) one split where $X$ has not been generated as a candidate and we are not able to calculate its exact projection counting on $\mathcal{D}$.

The first case does not need more discussion, since we have collected all the necessary information for calculating the entropy of $X$ on $\mathcal{D}$. The second case is more difficult since $X$ might be the miki but we cannot be sure, due to lack of information about its local entropy on (at least) one split. Therefore, we need to check the entropy of $X$ on $\mathcal{D}$ with a second MapReduce job intended to calculate its exact projection counting. The goal of this second round is to check that no local candidate has been ignored at the global scale. At the end of this round, we have the entropy of all the promising candidate itemsets and we are able to pick the one with the highest entropy. This is the architecture of our approach, the raw version of which (without optimization) is called Simple-PHIKS. So far, we have designed a distributed architecture and a miki extraction algorithm that, in our experiments reported in Section 6 outperforms ForwardSelection by several orders of magnitude. However, by exploiting and improving some concepts of information theory, we may significantly optimize this algorithm and further accelerate its execution at different parts of the architecture, as explained in the following sections.

\subsection{Candidate Reduction Using Entropy Upper Bound}

One of the shortcomings of the basic version of our two rounds approach is that the number of candidate itemsets, which should be processed in the second job, may be 
high for large databases as it will be illustrated by our experiments in Section 6 . This is particularly the case when the features are not uniformly distributed in the splits of mappers. These candidate itemsets are sent partially by the mappers (i.e., not by all of them), thus we cannot compute their total entropy in the corresponding reducer. This is why, in the basic version of our approach, we compute their entropy in the second job by reading again the database.

Here, we propose an efficient technique for significantly reducing the number of candidates. The main idea is to compute an upper bound for the entropy of the partially sent itemsets, and discard them if they have no chance to be a global miki. For this, we exploit the available information about the miki candidates sent by the mappers to the corresponding reducer.

Let us describe formally our approach. Let $X$ be a partially sent itemset, and $m$ be a mapper that has not sent $X$ and its projection frequencies to the reducer $R$ that is responsible for computing the entropy of $X$. In the reducer $R$, the frequency of $X$ projections for a part of the database is missing, i.e., in the split of $m$. We call these frequencies as missing frequencies. We compute an upper bound for the entropy of $X$ by estimating its missing frequencies. This is done in two steps. Firstly, finding the biggest subset of $X$, say $Y$, for which all frequencies are available and secondly, distributing the frequencies of $Y$ among the projections of $X$ in such a way that the entropy of $X$ be the maximum.

\subsubsection{Step 1}

The idea behind the first step is that the frequencies of the projections of an itemset $X$ can be derived from the projections of its subsets. For example, suppose two itemsets $X=\{A, B, C, D\}$ and $Y=\{A, B\}$, then the frequency of the projection $p=(1,1)$ of $Y$ is equal to the sum of the following projections in $X: p_{1}=(1,1,0,0)$, $p_{2}=(1,1,0,1), p_{3}=(1,1,1,0)$ and $p_{4}=(1,1,1,1)$. The reason is that in all these four projections, the features $A$ and $B$ exist, thus the number of times that $p$ occurs in the database is equal to the total number of times that the four projections $p_{1}$ to $p_{4}$ occur. This is stated by the following lemma.

Lemma 5.1. Let the itemset $Y$ be a subset of the itemset $X$, i.e., $Y \subseteq X$. Then, the frequency of any projection $p$ of $Y$ is equal to the sum of the frequencies of all projections of $X$ which involve $p$.

Proof. The proof can be easily done as in the above discussion.

In Step 1, among the available subsets of itemset $X$, i.e., those for which we have all projection frequencies, we choose the one that has the highest size. The reason is that its intersection with $X$ is the highest, thus our estimated upper bound about the entropy of $X$ will be closer to the real one.

\subsubsection{Step 2}

let $Y$ be the biggest available subset of $X$ in reducer $R$. After choosing $Y$, we distribute the frequency of each projection $p$ of $Y$ among the projections of $X$ that are derived from $p$. There may be many ways to distribute the frequencies. For instance, in the example of Step 1, if the frequency of $p$ is 6 , then the number of combinations for distributing 6 among the four projections $p_{1}$ to $p_{4}$ is equal to the solutions which can be found for the following equation: $x_{1}+x_{2}+x_{3}+x_{4}=6$ when $x_{i} \geq 0$. In general, the number of ways for distributing a frequency $f$ among $n$ projections is equal to the 
number of solutions for the following equation:

$$
x_{1}+x_{2}+\ldots+x_{n}=f \text { for } x_{i} \geq 0
$$

Obviously, when $f$ is higher than $n$, there is a lot of solutions for this equation. Among all these solutions, we choose a solution that maximizes the entropy of $X$. The following lemma shows how to choose such a solution.

Lemma 5.2. Let $\mathcal{D}$ be a database, and $X$ be an itemset. Then, the entropy of $X$ over $\mathcal{D}$ is the maximum if the possible projections of $X$ over $\mathcal{D}$ have the same frequency.

Proof. The proof is done by implying the fact that in the entropy definition (see Definition 2), the maximum entropy is for the case where all possible combinations have the same probability. Since, the probability is proportional to the frequency, then the maximum entropy is obtained in the case where the frequencies are the same.

The above lemma proposes that for finding an upper bound for the entropy of $X$ (i.e., finding its maximal possible entropy), we should distribute equally (or almost equally) the frequency of each projection in $Y$ among the derived projections in $X$. Let $f$ be the frequency of a projection in $Y$ and $n$ be the number of its derived projections, if ( $f$ modulo $n)=0$ then we distribute equally the frequency, otherwise we first distribute the quotient among the projections, and then the rest randomly.

After computing the upper bound for entropy of $X$, we compare it with the maximum entropy of the itemsets for which we have received all projections (so we know their real entropy), and discard $X$ if its upper bound is less than the maximum found entropy until now.

\subsection{Prefix/Suffix}

When calculating the local miki on a mapper, at each step we consider a set of candidates having size $j$ that share a prefix of size $j-1$. For instance, with the database of Table 2 and the subset of split $S_{1}$, the corresponding mapper will extract $(A, B)$ as the miki of size 2 . Then, it will build 3 candidates: $(A, B, C),(A, B, D)$ and $(A, B, E)$. A straightforward approach for calculating the joint entropy of these candidates would be to calculate their projection counting by means of an exhaustive scan over the data of $S_{1}$ (i.e., read the first transaction of $S_{1}$, compare it to each candidate in order to find their projections, and move to the next transaction). However, these candidates share a prefix of size 2: $(A, B)$. Therefore, we store the candidates in a structure that contains the prefix itemset, of size $j-1$, and the set of $|\mathcal{F}-j|$ suffix features. Then, for a transaction $T$, we only need to i) calculate $\operatorname{proj}(p, T)$ where $p$ is the prefix and ii) for each suffix feature $f$, find the projection of $f$ on $T$, append $\operatorname{proj}(f, T)$ to $\operatorname{proj}(p, T)$ and emit the result. Let us illustrate this principle with the example above (i.e., first transaction of $S_{1}$ in Table 2. The structure is as follows: $\{$ prefix $=(A, B)$ :suffixes $=C, D, E\}$. With this structure, instead of comparing $(A, B, C),(A, B, D)$ and $(A, B, E)$ to the transaction and find their respective projections, we calculate the projection of $(A, B)$, their prefix, i.e., $(0,0)$, and the projection of each suffix, i.e., (1), (0) and (0) for $C, D$, and $E$ respectively. Each suffix projection is then added to the prefix projection and emitted. In our case, we build three projections: $(0,0,1),(0,0,0)$ and $(0,0,0)$, and the mapper will emit $((A, B, C):(0,0,1)),((A, B, D):(0,0,0))$ and $((A, B, E):(0,0,0))$. 


\subsection{Incremental Entropy Computation in Mappers}

In the basic version of our two rounds approach, each mapper performs many scans over its split to compute the entropy of candidates and finally find the local miki. Given $k$ as the size of the requested itemset, in each step $j$ of the $k$ steps in the local miki algorithm, the mapper uses the itemset of size $j-1$ discovered so far, and builds $|F|-j$ candidate itemsets before selecting the one having the highest entropy. For calculating each joint entropy, a scan of the input split is needed in order to compute the frequency (and thus the probability) of projections. Let $|F|$ be the number of features in the database, then the number of scans done by each mapper is $O(k *|F|)$. Although the input split is kept in memory, this high number of scans over the split is responsible for the main part of the time taken by the mappers.

In this Section, we propose an efficient approach to significantly reduce the number of scans. Our approach that incrementally computes the joint entropies, needs to do in each step just one scan of the input split. Thus, the number of scans done by this approach is $O(k)$.

To incrementally compute the entropy, our approach takes advantage of the following lemma.

Lemma 5.3. Let $X$ be an itemset, and suppose we make an itemset $Y$ by adding a new feature $i$ to $X$, i.e., $Y=X+\{i\}$. Then, for each projection $p$ in $X$ two projections $p_{1}=p .0$, and $p_{2}=p .1$ are generated in $Y$, and the sum of the frequency of $p_{1}$ and $p_{2}$ is equal to that of $p$, i.e., $f(p)=f\left(p_{1}\right)+f\left(p_{2}\right)$.

proof. The projections of $Y$ can be divided into two groups: 1) those that represent transactions containing $i ; 2$ ) those representing the transactions that do not involve $i$. For each projection $p_{1}$ in the first group, there is a projection $p_{2}$ in the second group, such that $p_{1}$ and $p_{2}$ differ only in one bit, i.e., the bit that represents the feature $i$. If we remove this bit from $p_{1}$ or $p_{2}$, then we obtain a projection in $X$, say $p$, that represents all transactions that are represented by $p_{1}$ or $p_{2}$. Thus, for each project $p$ in $X$, there are two projections $p_{1}$ and $p_{2}$ in $Y$ generated from $p$ by adding one additional bit, and the frequency of $p$ is equal to the sum of the frequencies of $p_{1}$ and $p_{2}$.

Our incremental approach for miki computing proceeds as follows. Let $X$ be the miki in step $j$. Initially, we set $X=\{\}$, with a null projection whose frequency is equal to $n$, i.e., the size of the database. Then, in each step $j(1 \leq j \leq k)$, we do as follows. For each remaining feature $i \in F-X$, we create a hash map $h_{i, j}$ containing all projections of the itemset $X+\{i\}$, and we initiate the frequency of each projection to zero. Then, we scan the set of transactions in the input split of the mapper. For each transaction $t$, we obtain a set $S$ that is the intersection of $t$ and $F-X$, i.e., $S=t \cap(F-X)$. For each feature $i \in S$, we obtain the projection of $t$ over $X+\{i\}$, say $p_{2}$, and increment by one the frequency of the projection $p_{2}$ in the hash map $h_{i, j}$. After scanning all transactions of the split, we obtain the frequency of all projections ending with 1 . For computing the projections ending with 0 , we use Lemma 5.3 as follows. Let $p .0$ be a projection ending with 0 , we find the projection $p .1$ (i.e., the projection that differs only in the last bit), and set the frequency of $p .0$ equal to the frequency of $p$ minus that of $p .1$, i.e., $f(p .0)=f(p)-f(p .1)$. By this way, we compute the frequency of projections ending with 0 .

After computing the frequencies, we can compute the entropy of itemset $X+\{i\}$, for each feature $i \in F-X$. At the end of each step, we add to $X$ the feature $i$ whose joint entropy with $X$ is the highest. We keep the hash map of the selected itemset, and remove all other hash maps including that of the previous step. Then, we go to the next step until finishing step $k$. Notice that to obtain the frequency of $p$ in step $j$, we use the 
hash map of the previous step, i.e., $H_{i, j-1}$, this is why, at each step we keep the hash map of the selected miki.

Let us now prove the correctness of our approach using the following Theorem.

Theorem 5.4. Given a database $\mathcal{D}$, and a value $k$ as the size of requested miki. Then, our incremental approach computes correctly the entropy of the candidate itemsets in all steps.

proof. To prove the correctness of our approach, it is sufficient to show that in each step the projection frequencies of $X+\{i\}$ are computed correctly. We show this by induction on the number of steps, i.e., $j$ for $1 \leq j \leq k$.

Base. In the first step, the itemset $X+\{i\}=\{i\}$ because initially $X=\{\}$. There are two projections for $\{i\}: p_{1}=(0)$ and $p_{2}=(1)$. The frequency of $p_{2}$ is equal to the number of transactions containing $i$. Thus during the scan of the split, we correctly set the frequency of $p_{2}$. Since there is no other projection for $i$, the frequency of $p_{1}$ is equal to $n-f\left(p_{2}\right)$, where $n$ is the size of the database. This frequency is found correctly by our approach. Thus, for step $j=1$ our approach finds correctly the projection frequencies of $X+\{i\}$.

Induction. we assume that our approach works correctly in step $j-1$, then we prove that it will work correctly in step $j$. The proof can be done easily by using Lemma 5.3 According this lemma, for each projection $p$ in step $j-1$ there are two projections $p_{1}=(p .0)$, and $p_{2}=(p .1)$ in step $j$. The frequency of $p_{2}$ is computed correctly during the scan of the split. We assume that the frequency of $p$ has been correctly computed in step $j-1$. Then, Lemma 5.3 implies that the frequency of $p_{1}$ has been also well computed since we have $f(p)=f\left(p_{1}\right)+f\left(p_{2}\right)$.

\subsection{Complete Approach}

Our approach depicts the core mining process of Parallel Highly Informative $K$-itemSet Algorithm (PHIKS). The major steps of PHIKS algorithm for miki discovery are summarized in Algorithms 1 and 2. Algorithm 1 depicts the mining process of the first MapReduce job of PHIKS, while Algorithm 2 depicts the mining process of its second MapReduce job. 


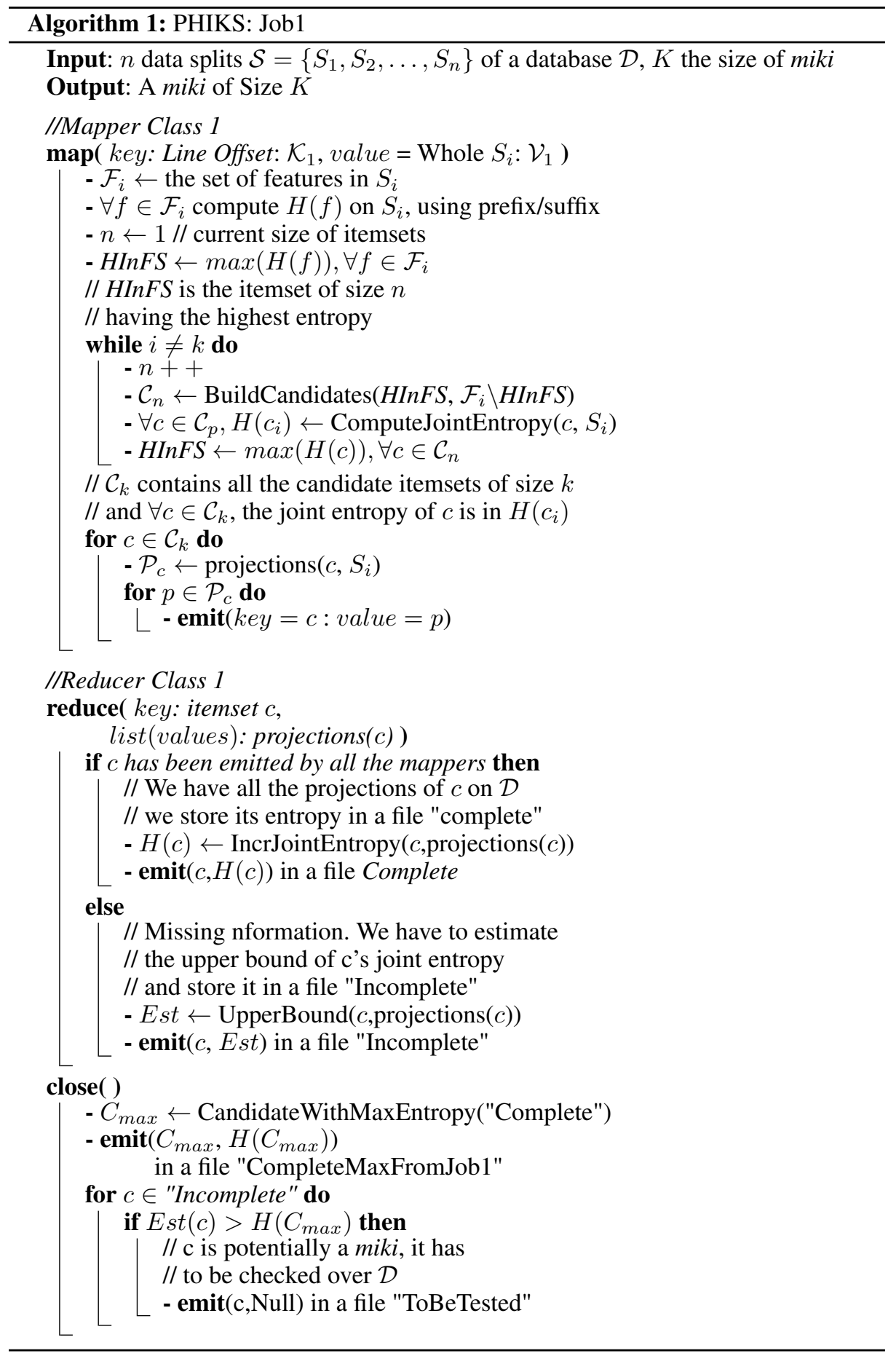




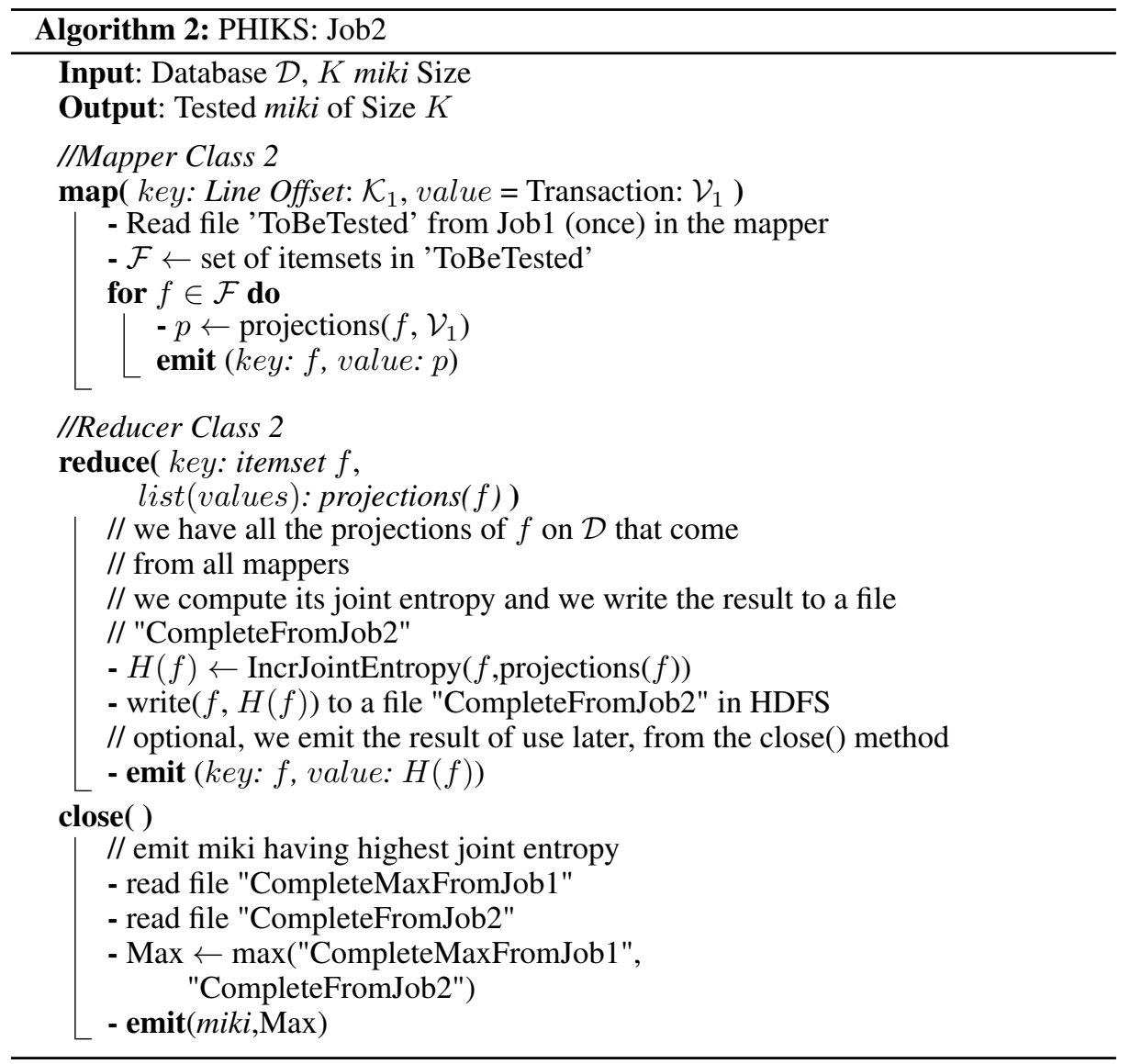

\section{Experiments}

To evaluate the performance of PHIKS, we have carried out extensive experimental tests. In Section 6.1, we depict our experimental setup and its main configurations. In Section 6.2 we depict the different used data sets in our various experiments. Lastly, in Section 6.3 we thoroughly analyze and investigate our different experimental results.

\subsection{Experimental Setup}

We implemented PHIKS algorithm on top of Hadoop-MapReduce using Java programming language version 1.7 and Hadoop (White, 2012) version 1.0.3. For comparison, we implemented a parallel version of Forward Selection (Knobbe and Ho, 2006) algorithm. To specify each presented algorithm, we adopt the notations as follow. We denote by 'PFWS' a parallel implementation of Forward Selection algorithm, by 'SimplePHIKS' an implementation of our basic two rounds algorithm without any optimization, and by 'Prefix' an extended version of Simple-PHIKS algorithm that uses the Prefix/Suffix method for accelerating the computations of the projection values. We denote by 'Upper-B' a version of our algorithm that reduces the number of candidates by esti- 


\begin{tabular}{cccc}
\hline Data Set & \# of Transactions & \# of Items & Size \\
\hline \hline Amazon Reviews & 34 millions & 31721 & 34 Gigabyte \\
English Wikipedia & 5 millions & 23805 & 49 Gigabytes \\
ClueWeb & 632 millions & 141826 & 1 Terabyte \\
\hline
\end{tabular}

Table 3. Data Sets Description

mating the joint entropies of miki based on an upper bound joint entropy. We denote by 'Upper-B-Prefix' an extended version of Upper-B algorithm that employs the technique of prefix/suffix. Lastly, we denote by 'PHIKS' an improved version of Upper-B-Prefix algorithm that uses the method of incremental entropy for reducing the number of data split scans at each mapper.

We carried out all our experiments on the Grid5000 (Grid5000, n.d.) platform, which is a platform for large-scale data processing. In our experiments, we have used clusters of 16 and 48 nodes respectively for Amazon Reviews, Wikipedia data sets and ClueWeb data set. Each machine is equipped with Linux operating system, 64 Gigabytes of main memory, Intel Xeon X3440 4 core CPUs and 320 Gigabytes SATA hard disk.

In our experiments, we measured three metrics: 1) the response time of the compared algorithms, which is the time difference between the beginning and the end of a maximally informative $k$-itemsets mining process; 2 ) the quantity of transferred data (i.e., between the mappers and the reducers) of each maximally informative $k$-itemsets mining process; 3 ) the energy consumption for each maximally informative $k$-itemsets mining process. To this end, we used the metrology API and Ganglia infrastructure of the Grid5000 platform that allow to measure the energy consumption of the nodes during an experiment.

Basically, in our experiments, we consider the different performance measurements when the size $k$ of the itemset (miki to be discovered) is high.

\subsection{Data Sets}

To better evaluate the performance of PHIKS algorithm, we used three real-world data sets as described in Table 3. The first one is the whole 2013 Amazon Reviews data set (Amazon, n.d.), having a total size of 34 Gigabytes and composed of 35 million reviews. The second data set is the 2014 English Wikipedia data set (English Wikipedia Articles, 2014), having a total size of 49 Gigabytes and composed of 5 million articles. The third data set is a sample of ClueWeb English data set (The ClueWeb09 Dataset, 2009) with size of around one Terabyte and having 632 million articles. For English Wikipedia and ClueWeb data sets, we performed a data cleaning task; we removed all English stop words from all articles, and obtained data sets where each article represents a transaction (features, items, or attributes are the corresponding words in the article). Likewise, for Amazon Reviews data set, we removed all English stop words from all reviews. Each review represents a transaction in our experiments on Amazon Reviews data set.

\subsection{Results}

In this Section, we report the results of our experimental evaluation. 


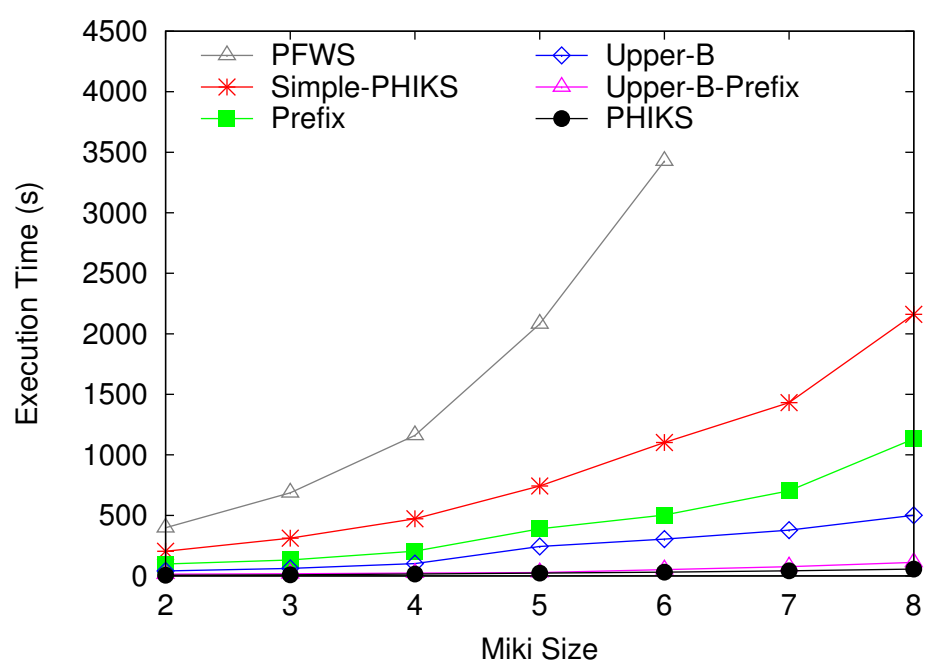

(a) All algorithms

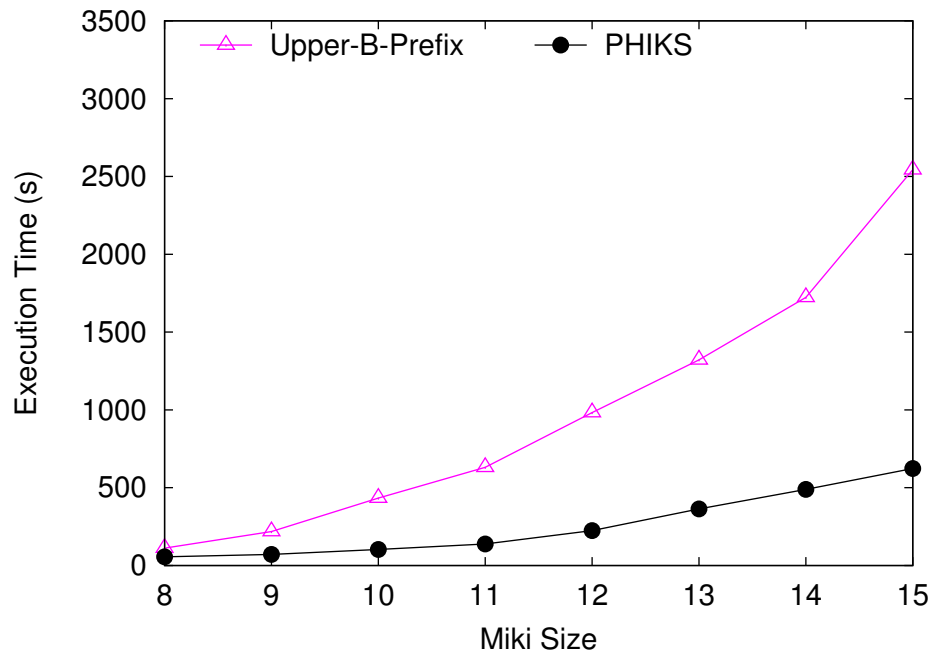

(b) Focus on scalable algorithms

Fig. 1. Runtime and scalability on Amazon Reviews Data Set

\subsubsection{Runtime and Scalability}

Figures 1, 2, and 3 show the results of our experiments on Amazon Reviews, English Wikipedia and ClueWeb data sets. Figures 1(a) and 1(b) give an overview on our experiments on the Amazon Reviews data set. Figure 1(a) illustrates the the performance of different algorithms when varying the itemset sizes from 2 to 8 . We see that the response time of Forward Selection algorithm (PFWS) grows exponentially and gets quickly very high compared to other algorithms. Above a size $k=6$ of itemsets, PFWS cannot continue scaling. This is due to the multiple database scans that it performs to determine an 


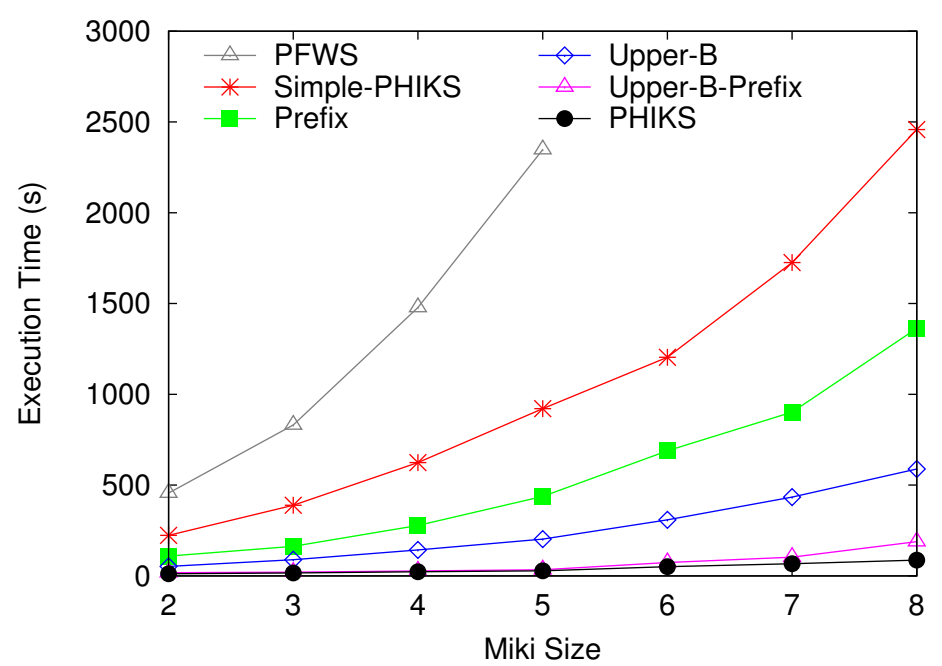

(a) All algorithms

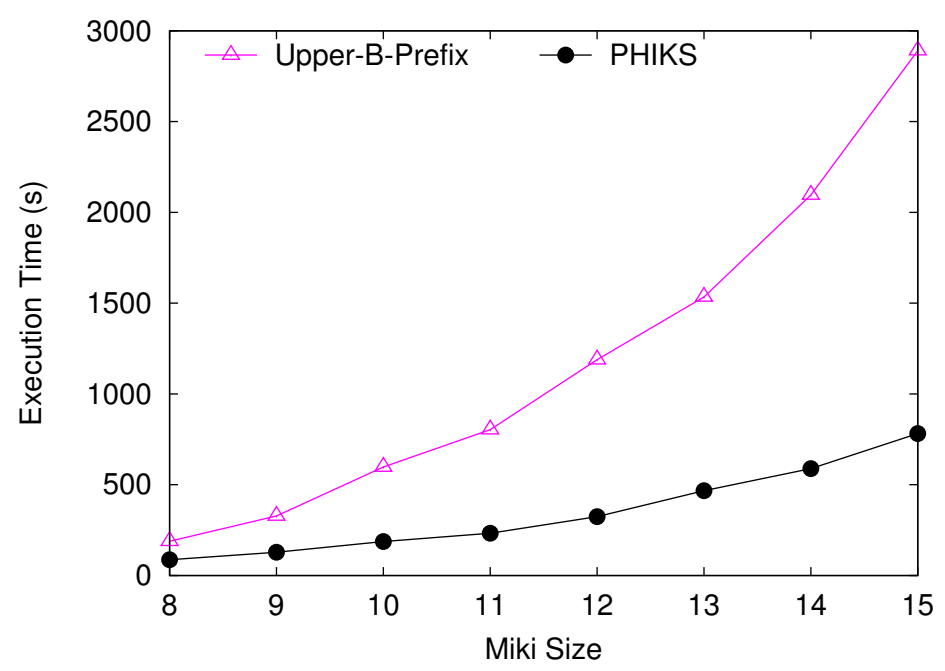

(b) Focus on scalable algorithms

Fig. 2. Runtime and scalability on English Wikipedia Data Set

itemset of size $k$ (i.e, PFWS needs to perform $k$ MapReduce jobs). In the other hand, the performance of Simple-PHIKS algorithm is better than PFWS; it continues scaling with higher $k$ values. This difference in the performance between the two algorithms illustrates the significant impact of mining itemsets in the two rounds architecture.

Moreover, by using further optimizing techniques, we clearly see the improvements in the performance. In particular, with an itemset having size $k=8$, we observe a good performance behavior of Prefix comparing to Simple-PHIKS. This performance gain in the runtime reflects the efficient usage of Prefix/Suffix technique for speeding up miki parallel extraction. Interestingly, by estimating miki at the first MapReduce job, we 


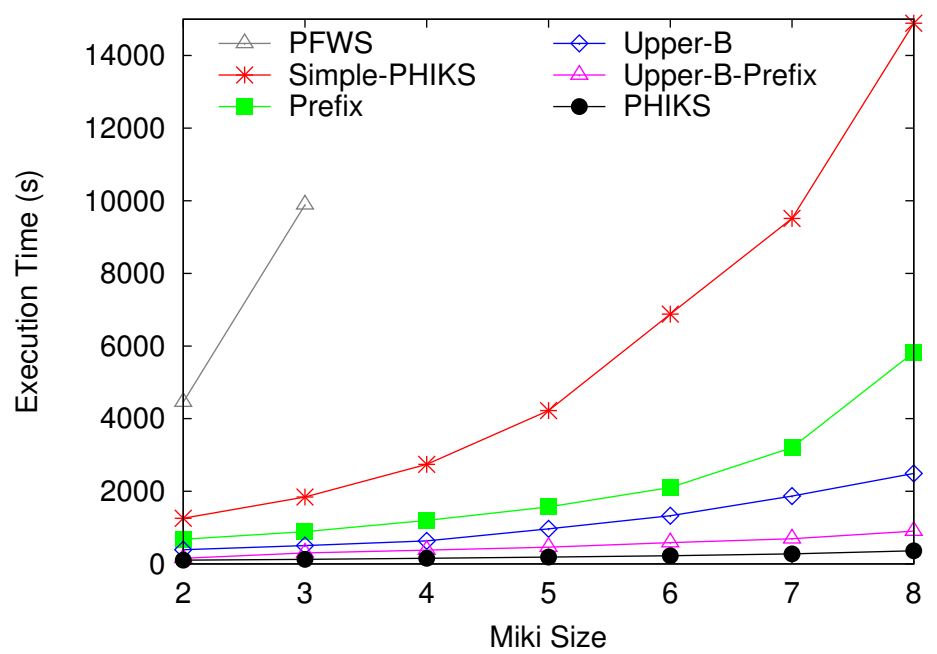

(a) All algorithms

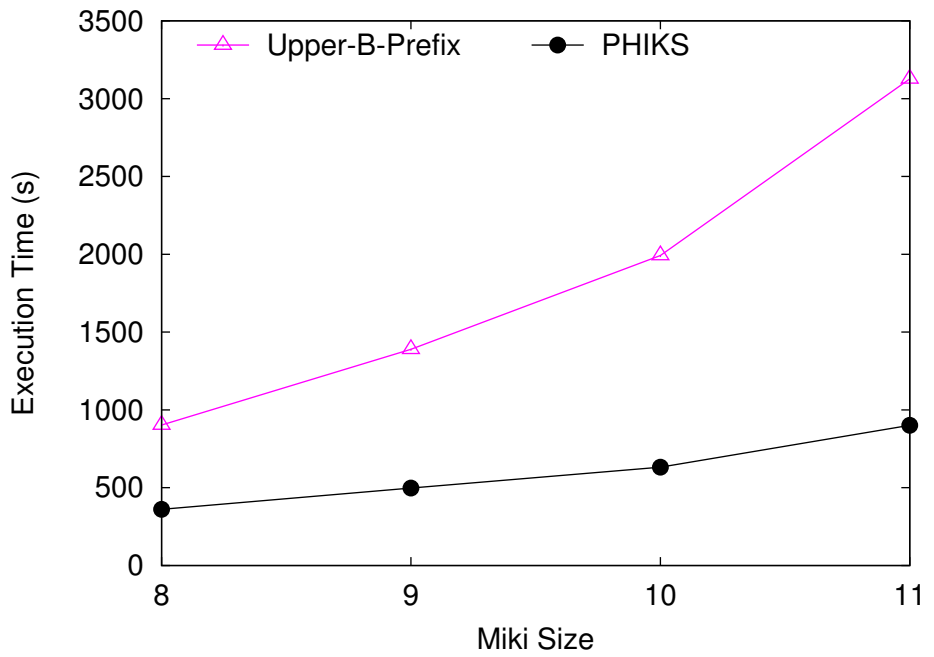

(b) Focus on scalable algorithms

Fig. 3. Runtime and scalability on ClueWeb Data Set

record a very good response time as shown by Upper-B algorithm. In particular, with $k=8$ we see that Upper-B algorithm roughly outperforms Simple-PHIKS by a factor of 3. By coupling the Prefix/Suffix technique with Upper-B algorithm, we see very good improvements in the response time, which is achieved by Upper-B-Prefix. Finally, by taking advantage of our incremental entropy technique for reducing the number of data split scans, we record an outstanding improvement in the response time, as shown by PHIKS algorithm.

Figure 1(b) highlights the difference between the algorithms that scale in Figure 1(a) Although Upper-B-Prefix continues to scale with $k=8$, it is outperformed by 
PHIKS algorithm. With itemsets of size $k=15$, we clearly observe a big difference in the response time between Upper-B-Prefix and PHIKS. The significant performance of PHIKS algorithm illustrates its robust and efficient core mining process.

Figures 2(a) and 2(b) report our experiments on the English Wikipedia data set. Figure 2(a) gives a complete view on the the performance of different presented algorithms when varying the itemset sizes from 2 to 8 . Similarly as in Figure 1(a) in Figure 2(a) we clearly see that the execution time of Forward Selection algorithm (PFWS) is very high compared to other presented alternatives. When the itemsets size reach values greater than $k=5$, PFWS stops scaling. In the other side, we observe that Simple-PHIKS algorithm continues scaling and gives better performance than PFWS.

Performing more optimization, we significantly speed up the miki extraction. Specifically, with itemsets size $k=8$, we see that the performance of Prefix is better than Simple-PHIKS. This difference in the performance behavior between the two algorithms explains the high impact of using Prefix/Suffix technique to speed up the whole mining process of the parallel miki extraction. By going on for further optimization using our efficient heuristic technique for estimating miki at the first MapReduce job, we get a significant improvement in the execution time as shown by Upper-B algorithm. Particularly, with itemsets size $k=8$ we clearly see that Upper-B algorithm performance is better than Simple-PHIKS. By using Prefix/Suffix technique with Upper-B algorithm, we record a significant improvement in the performance as shown by UpperB-Prefix. Eventually, based on our efficient technique of incremental entropy, we record a very significant performance improvement as shown by PHIKS algorithm.

Figure 2(b) illustrates the difference between the algorithms that scale in Figure 2(a) Despite the scalability recorded by Upper-B-Prefix when $k=8$, Upper-B-Prefix gives very less performance compared to PHIKS algorithm. In particular, with higher itemsets size (e.g., $k=15$ ), we record a large difference in the execution time between UpperB-Prefix and PHIKS algorithms. This difference in the performance between the two algorithms reflects the efficient and robust core mining process of PHIKS algorithm. In Figures 3(a) and 3(b), similar experiments have been conducted on the ClueWeb data set. We observe that the same order between all algorithms is kept compared to Figures 1(a), 1(b), 2(a) and 2(b) In particular, we see that PFWS algorithm suffers from the same limitations as could be observed on the Amazon Reviews and Wikipedia data sets in Figure 1(a) and Figure 2(a). With an itemset size of $k=8$, we clearly observe a significant difference between PHIKS algorithm performance and all other presented alternatives. This difference in the performance is better illustrated in Figure 3(b) By increasing the size $k$ of miki from 8 to 11, we observe a very good performance of PHIKS algorithm. Although, Upper-B-Prefix algorithm scales with $k=11$, it is outperformed by PHIKS.

\subsection{2. miki Candidates Pruning}

Figure 4 gives a complete overview on the total number of miki candidates being tested at the second MapReduce job for both Simple-PHIKS and PHIKS algorithms. Figure 4(a) illustrates the number of miki candidates being validated at the first MapReduce job on the Wikipedia data set. By varying the parameter size $k$ of itemsets from 2 to 8 , we observe a significant difference in the number of miki candidates being sent by each algorithm to its second MapReduce job. With $k=8$, Simple-PHIKS algorithm sends to its second job roughly 6 times more candidates than PHIKS. This important reduction in the number of candidates to be tested in the second job is achieved due to our efficient technique for estimating the joint entropies of miki with very low upper bounds. Likewise, in Figure 4(b), we record a very good performance of PHIKS comparing to 


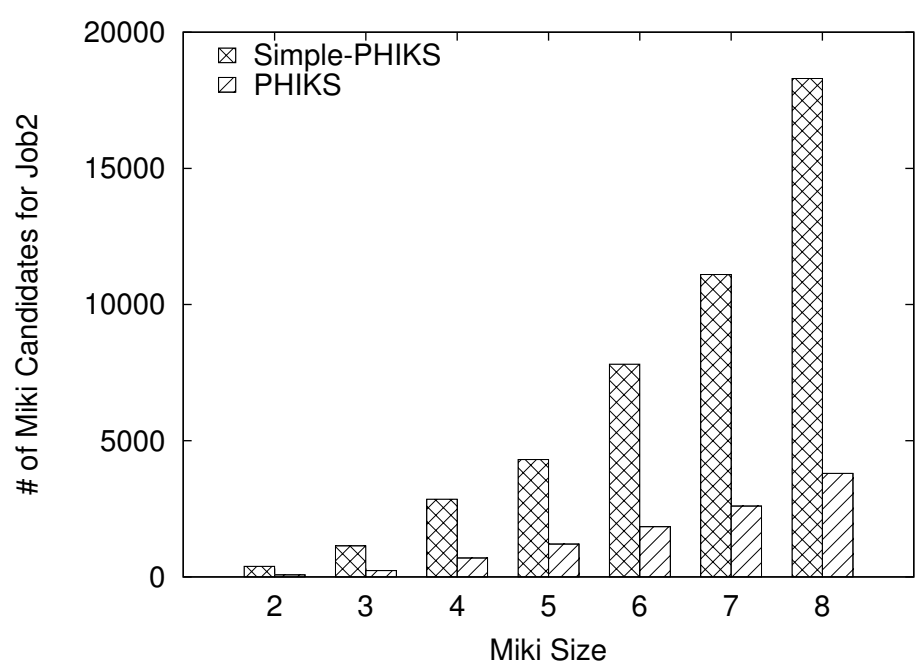

(a) Wikipedia data set

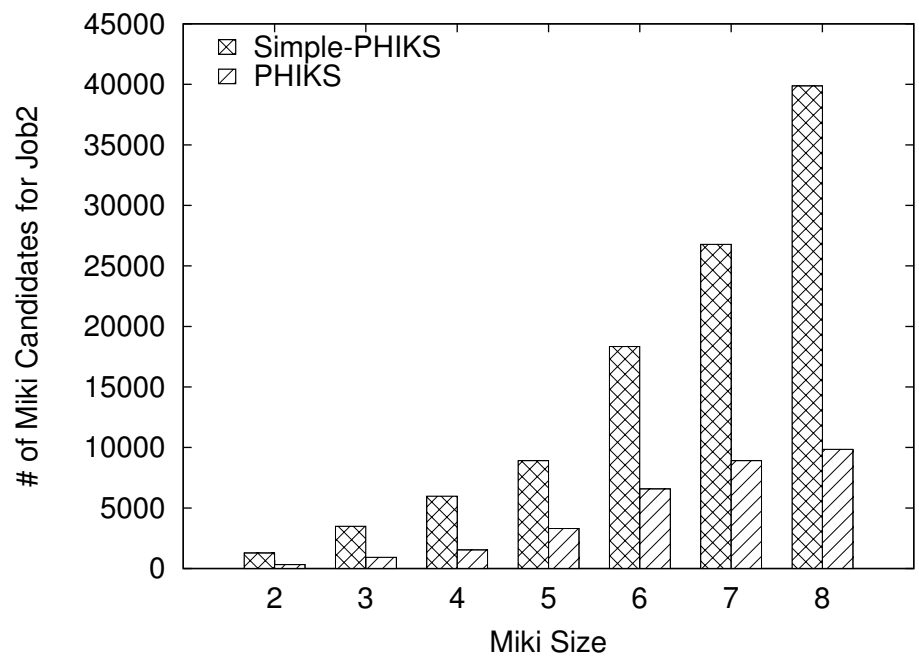

(b) ClueWeb data set

Fig. 4. Candidate Pruning

Simple-PHIKS. This outstanding performance of Simple-PHIKS algorithm reflects its high capability and its effectiveness for a very fast and successful miki extraction.

\subsubsection{Data Communication and Energy Consumption}

Figure 5 gives an entire view of the quantity of transferred data (in megabyte) over the network by each presented algorithm on the three data sets. Respectively Figures 5(a) 5(b) and 5(c) show the performance of each presented maximally informative $k$-itemsets mining process on Amazon Reviews, English Wikipedia and ClueWeb data sets. In all 


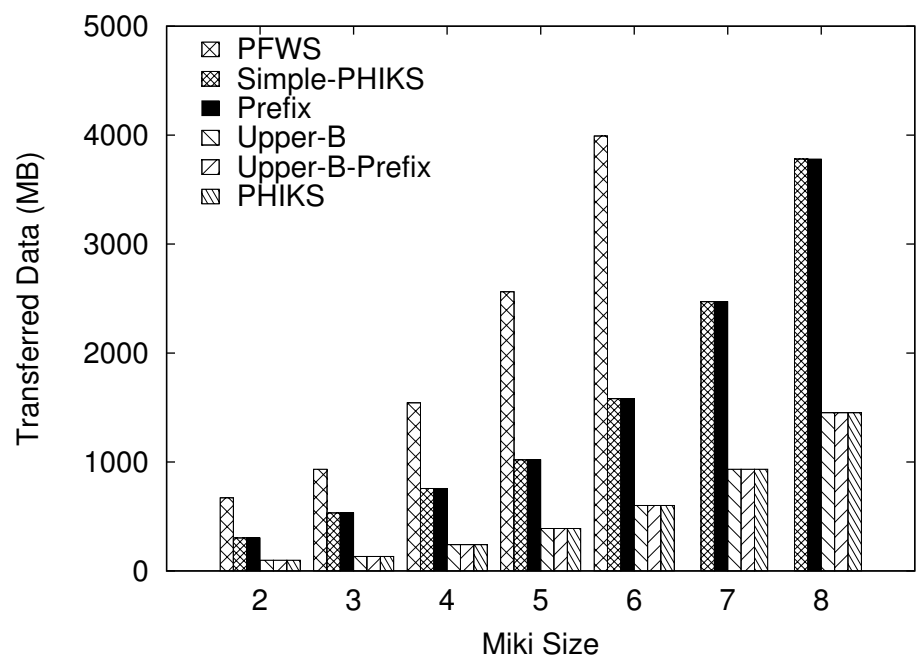

(a) Amazon Reviews data set

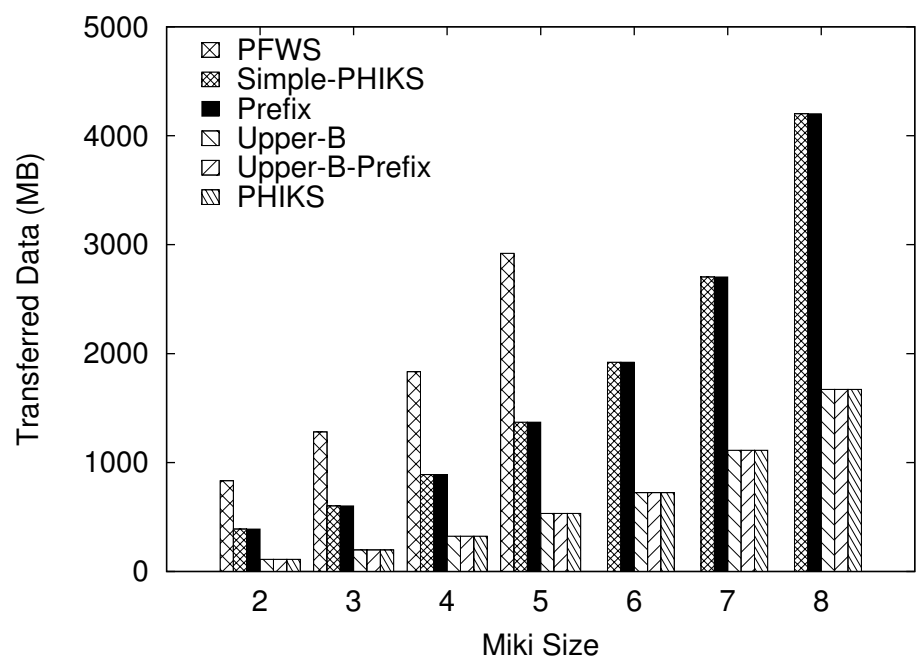

(b) Wikipedia data set

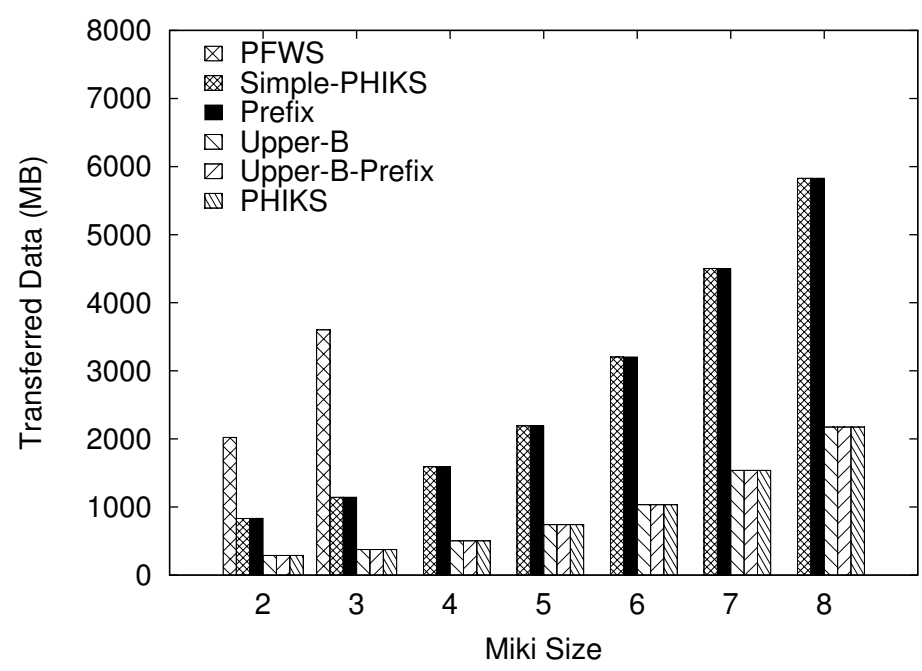

(c) ClueWeb data set

Fig. 5. Data Communication 


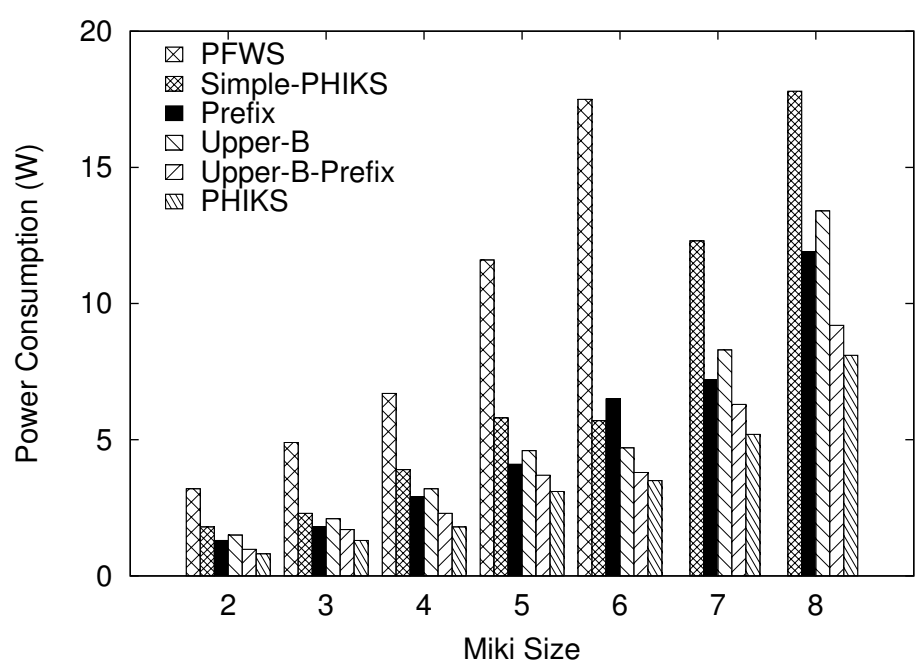

(a) Amazon Reviews data set

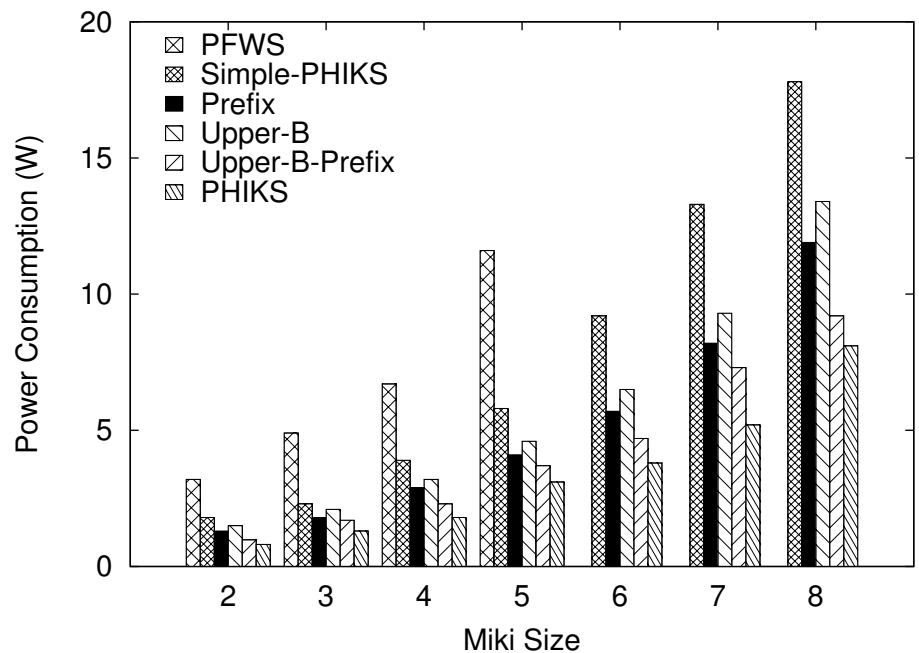

(b) Wikipedia data set

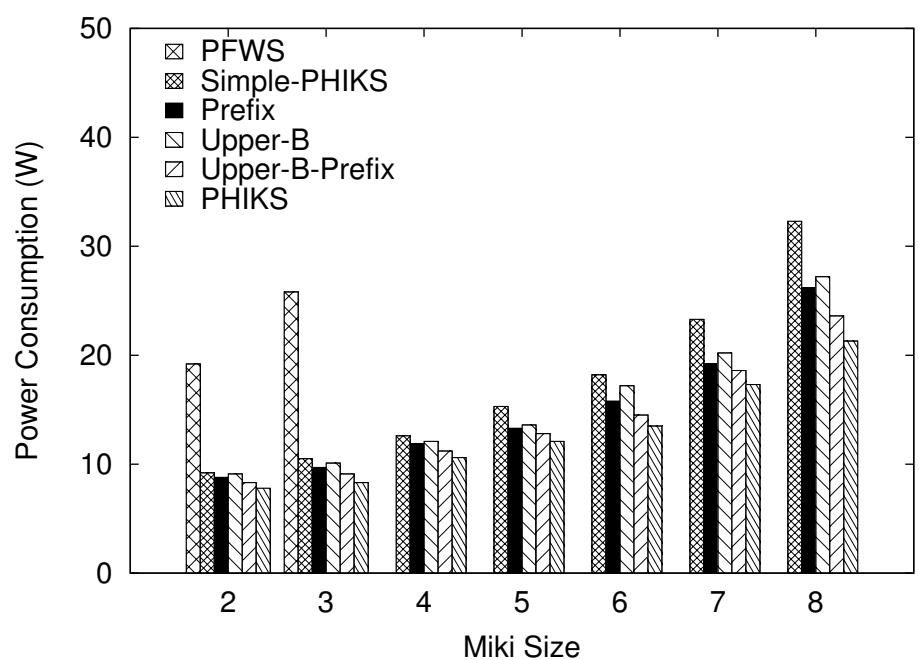

(c) ClueWeb data set

Fig. 6. Energy Consumption 
figures, we observe that PFWS algorithm has the highest peak. This is due to its multiple MapReduce jobs executions. In the other hand, we see that Simple-PHIKS and Prefix algorithms have smaller peaks. This is because Simple-PHIKS and its optimized Prefix version algorithm (for fast computation of the local entropies at the mappers) rely on two MapReduce jobs whatever the miki size to be discovered. We see that Upper-B, UpperB-Prefix and PHIKS outperform all other presented algorithms in terms of transferred data. This is due to the impact of estimating the joint entropies at their first MapReduce job which reduces the number of miki candidates (i.e., data) being tested at their second MapReduce job.

We also measured the energy consumption (in Watt) of the compared algorithms during their execution. We used the Grid5000 (Grid5000, n.d.) tools that measure the power consumption of the nodes during a job execution. Figure 6 shows the total amount of the power consumption of each presented maximally informative $k$-itemsets mining process on Amazon Reviews, English Wikipedia and ClueWeb data sets. In Figures 6(a) 6(b) and 6(c) we observe that the energy consumption increases when increasing the size $k$ of the miki to be discovered for each algorithm. We see that PHIKS still gives a lower consumption comparing to other presented algorithms. This is simply due to the higher optimizations in its core mining process. Actuelly the smaller number of candidates being tested during the second MapReduce job of PHIKS calls for a lower number of I/O access when computing the entropies. All of these different factors make PHIKS consumes less energy compared to other presented algorithms.

\section{Conclusion}

In this paper, we proposed a reliable and efficient MapReduce based parallel maximally informative $k$-itemset algorithm namely PHIKS that has shown significant efficiency in terms of runtime, communication cost and energy consumption. PHIKS elegantly determines the miki in very large databases with at most two rounds. With PHIKS, we propose a bunch of optimizing techniques that renders the miki mining process very fast. These techniques concern the architecture at a global scale, but also the computation of entropy on distributed nodes, at a local scale. The result is a fast and efficient discovery of miki with high itemset size. Such ability to use high itemset size is mandatory when dealing with Big Data and particularly one Terabyte like what we have done in our experiments. Our results show that PHIKS algorithm outperforms other alternatives by several orders of magnitude, and makes the difference between an inoperative and a successful miki extraction.

As a future work, we plan to apply our technique of extracting miki to handle the problem of text classification. By using our PHIKS approach, we strongly believe that the extracted miki would highly discriminate the database, which is in turn would result in high classification accuracy and fast learning process.

Acknowledgements. Experiments presented in this paper were carried out using the Grid'5000 experimental testbed, being developed under the INRIA ALADDIN development action with support from CNRS, RENATER and several universities as well as other funding bodies (see https://www.grid5000.fr).

\section{References}

Agrawal, R. and Srikant, R. (1994), Fast algorithms for mining association rules in large databases, in 'Proceedings of International Conference on Very Large Data Bases (VLDB)', pp. 487-499. 
Amazon (n.d.), http://snap.stanford.edu/data/web-Amazon-links.html

Anand, R. (2012), Mining of massive datasets, Cambridge University Press, New York, N.Y. Cambridge.

Berberich, K. and Bedathur, S. (2013), Computing n-gram statistics in mapreduce, in 'Proceedings of the 16th International Conference on Extending Database Technology (EDBT)', pp. 101-112.

Berry, M. W. and Castellanos, M. (n.d.), Survey of Text Mining II: Clustering, Classification, and Retrieval, 1 edn.

Bizer, C., Boncz, P. A., Brodie, M. L. and Erling, O. (2011), 'The meaningful use of big data: four perspectives - four challenges', SIGMOD Record 40(4), 56-60.

Brin, S., Motwani, R. and Silverstein, C. (1997), 'Beyond market baskets: Generalizing association rules to correlations', SIGMOD Rec. 26(2), 265-276. URL: http://doi.acm.org/10.1145/253262.253327

Chandrashekar, G. and Sahin, F. (2014), 'A survey on feature selection methods', Computers and Electrical Engineering 40(1), $16-28$.

Cover, T. M. (2006), Elements of information theory, Wiley-Interscience, Hoboken, N.J.

Dean, J. and Ghemawat, S. (2008), 'Mapreduce: simplified data processing on large clusters', Commun. ACM 51(1), 107-113.

English Wikipedia Articles (2014), http://dumps.wikimedia.org/enwiki/latest

Ghahramani, Z. (2004), Unsupervised learning, in 'Advanced Lectures on Machine Learning', pp. 72-112.

Giannella, C., Han, J., Pei, J., Yan, X. and Yu, P. S. (2002), 'Mining frequent patterns in data streams at multiple time granularities'.

Gray, R. (2011), Entropy and information theory, Springer, New York.

Greengrass, E. (2000), 'Information retrieval: A survey'.

Grid5000 (n.d.), https://www.grid5000.fr/mediawiki/index.php/Grid5000:Home

Guyon, I. and Elisseeff, A. (2003), 'An introduction to variable and feature selection', J. Mach. Learn. Res. 3, $1157-1182$

Hadoop (2014), http://hadoop.apache.org.

Han, J. (2012), Data mining : concepts and techniques, Elsevier/Morgan Kaufmann.

Han, Pei and Yin (2000), 'Mining frequent patterns without candidate generation', SIGMODREC: ACM SIGMOD Record 29.

Heikinheimo, H., Hinkkanen, E., Mannila, H., Mielikäinen, T. and Seppänen, J. K. (2007), Finding lowentropy sets and trees from binary data, in 'Proceedings of ACM SIGKDD International Conference on Knowledge Discovery and Data Mining (KDD)', pp. 350-359.

Herrera, F., Carmona, C., González, P. and del Jesus, M. (2011), 'An overview on subgroup discovery: foundations and applications', Knowledge and Information Systems 29(3), 495-525. URL: http://dx.doi.org/10.1007/s10115-010-0356-2

Knobbe, A. J. and Ho, E. K. Y. (2006), Maximally informative k-itemsets and their efficient discovery, in 'Proceedings of ACM SIGKDD International Conference on Knowledge Discovery and Data Mining (KDD)', pp. 237-244.

Kotsiantis, S. B. (2007), Supervised machine learning: A review of classification techniques, in 'Proceedings of International Conference on Emerging Artificial Intelligence Applications in Computer Engineering', pp. 3-24.

Li, H., Wang, Y., Zhang, D., Zhang, M. and Chang, E. Y. (2008), Pfp: parallel fp-growth for query recommendation, in 'Proceedings of the ACM Conf. on Recommender Systems (RecSys)', pp. 107-114.

Miliaraki, I., Berberich, K., Gemulla, R. and Zoupanos, S. (2013), Mind the gap: Large-scale frequent sequence mining, in 'Proceedings of the 2013 ACM SIGMOD International Conference on Management of Data (SIGMOD)', pp. 797-808.

Moens, S., Aksehirli, E. and Goethals, B. (2013), Frequent itemset mining for big data, in 'IEEE International Conference on Big Data', pp. 111-118.

Riondato, M., DeBrabant, J. A., Fonseca, R. and Upfal, E. (2012), Parma: a parallel randomized algorithm for approximate association rules mining in mapreduce, in '21st ACM International Conference on Information and Knowledge Management (CIKM)', pp. 85-94.

Savasere, A., Omiecinski, E. and Navathe, S. B. (1995), An efficient algorithm for mining association rules in large databases, in 'Proceedings of International Conference on Very Large Data Bases (VLDB)' pp. 432-444.

Tanbeer, S., Ahmed, C. and Jeong, B.-S. (2009), Parallel and distributed frequent pattern mining in large databases, in ' 11 th IEEE International Conference on High Performance Computing and Communications (HPCC)', pp. 407-414.

Tatti, N. (2010), Probably the best itemsets, in 'Proceedings of the 16th ACM SIGKDD International Conference on Knowledge Discovery and Data Mining, Washington, DC, USA, July 25-28, 2010', pp. 293-302. URL: http://doi.acm.org/10.1145/1835804.1835843 
Teng, W.-G., Chen, M.-S. and Yu, P. S. (2003), A regression-based temporal pattern mining scheme for data streams, in 'Proceedings of International Conference on Very Large Data Bases (VLDB)', pp. 93-104.

The ClueWeb09 Dataset (2009), http: / / www. lemurproject.org/clueweb09.php/

White, T. (2012), Hadoop : the definitive guide, O'Reilly.

Zaharia, M., Chowdhury, M., Franklin, M. J., Shenker, S. and Stoica, I. (2010), Spark: Cluster computing with working sets, in 'Proceedings of the 2Nd USENIX Conf. on Hot Topics in Cloud Computing', pp. 10-10.

Zhang, C. and Masseglia, F. (2010), Discovering highly informative feature sets from data streams, in 'Database and Expert Systems Applications', pp. 91-104. 